\title{
Numb Chin Syndrome Secondary to Vestibular Schwannoma
}

\author{
Nilofer Halim ${ }^{1 *}$, Chaithra Kalkur ${ }^{2}$, Anusha L Rangare ${ }^{2}$ and S Padmashree ${ }^{3}$ \\ ${ }^{1}$ Senior Lecturer, Department of Oral Medicine and Radiology, Century International Institute of Dental Sciences and Research Centre, India \\ ${ }^{2}$ Reader, Department of Oral Medicine and Radiology, Century International Institute of Dental Sciences and Research Centre, India \\ ${ }^{3}$ Professor and Head of Department, Department of Oral Medicine and Radiology, Vydehi Institute of Dental Sciences and Research Centre, \\ India
}

*Corresponding author: Nilofer Halim, Senior Lecturer, Department of Oral Medicine and Radiology, Century International Institute of Dental Sciences and Research Centre, India

\begin{abstract}
Numb chin syndrome (NCS) or mental neuropathy is a sensory neuropathy. It can present as trivial finding of hypoesthesia/ paraesthesia or pain over the chin and area supplied by the mandibular nerve. The etiology can vary from dental pathogenesis to malignancy and neurological disorders. Here we present a case report of 18 year old female patient, who presented with numb chin syndrome and on further investigation was detected with a large vestibular schwannoma.
\end{abstract}

Keywords: Numb Chin Syndrome; Schwannoma; Neurofibromatosis

Abbreviations: NCS: Numb Chin Syndrome; TMJ: Temporomandibular Joint; OPG: Orthopantomography; MRI: Magnetic Resonance Imaging; CP: Cerebellopontine; NF 1,2,3: Neurofibromatosis 1,2,3

\section{Introduction}

Parasthesia or Numbness over the chin is common symptom in dental practice. It is generally seen as adverse effect of peripheral neurectomy that may occur during oral surgical procedures involving inferior alveolar nerve. However, in certain instances it may be symptom of serious underlying disorder such as malignancy or neurological pathology. In this case report we highlight one such case of a young patient presenting with a trivial symptom of numbness over the chin which was the only manifestation of a large intracranial Schwannoma.

\section{Case Report}

An 18 year old female patient reported with chief complaint of numbness in the left lower side of the face for 2 months. Her medical and dental history was not significant. She was well built and nourished, conscious and cooperative during the examination. On extra oral examination there was no facial a symmetry. Temporomandibular examination was normal. A single jugulodigastric lymph node was enlarged and tender on the left side. It was firm in consistency and not fixed. On intraoral examination, two ulcers spherical in shape and less than $1 \mathrm{~cm}$ in diameter with peri inflammatory halo were noticed on the left lower labial mucosa. Tongue was coated with mild erythematous changes on the left lateral aspect, soft palate and tonsillar pillars on the left side were also erythematous. We performed cranial nerve examination and found deficit on the mandibular division of trigeminal nerve. Pulp vitality testing was done and all the teeth on the lower left quadrant did not respond. OPG showed widening of the mandibular foramen. A provisional diagnosis of numb chin syndrome was made, and patient was advised for a complete blood count and MRI of head and neck. Blood reports were within normal limits. The MRI detected a well-defined encapsulated heterogenous signal intensity in the left cerebellopontine angle (CP) which had solid and cystic components and measured $4.7 \mathrm{X} 4.3 \mathrm{~cm}$ with intracanalicular extension causing widening of internal carotid 
artery. Compression on the 4th ventricle, pons and left cerebellar hemisphere. Similar intensity lesion was noticed in carotid space in the left side of neck measuring 5 X $3.7 \mathrm{~cm}$ (Figures 1 \& 2). Patient was referred to neurologist and was diagnosed as schwannoma associated with neurofibromatosis type 2. She was referred to neurosurgeon, who has kept her under observation.

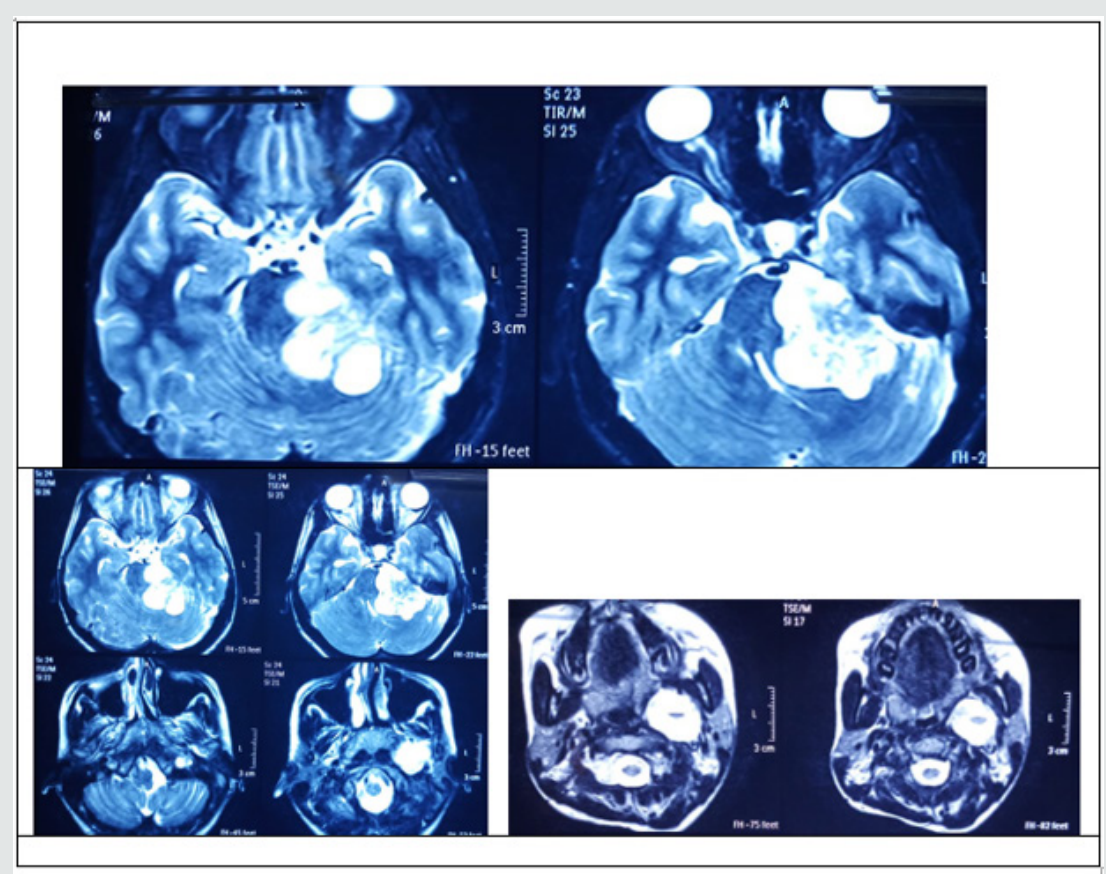

Figure 1: MRI Axial View.

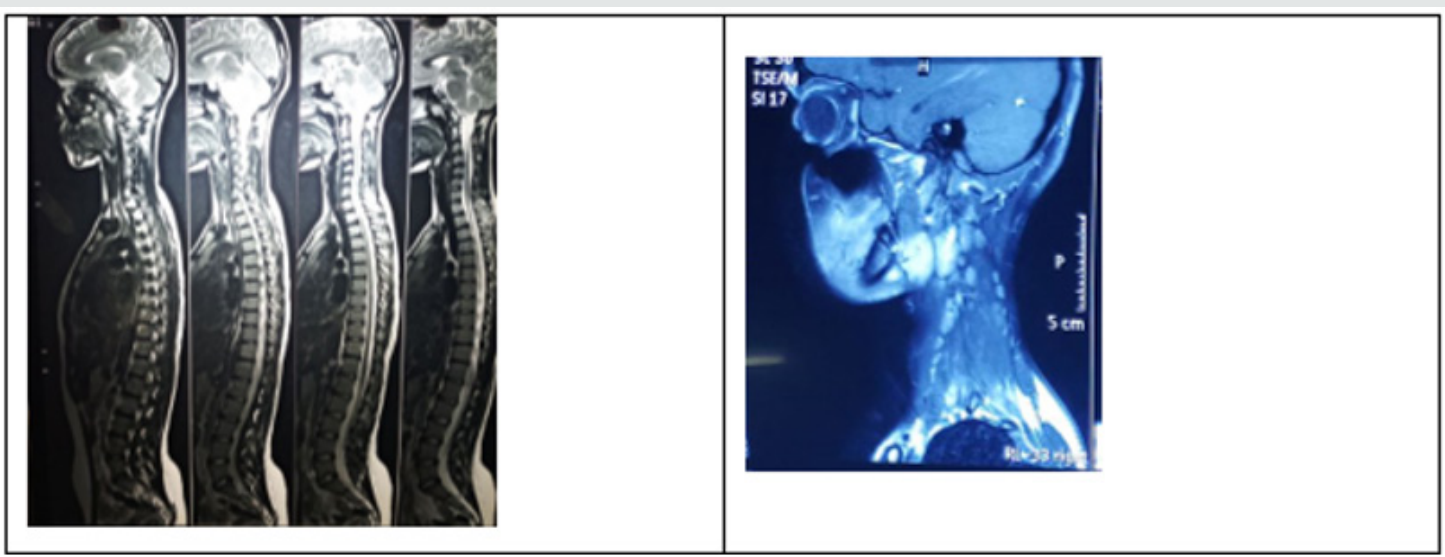

Figure 2: MRI Sagittal View.

\section{Discussion}

Numb chin syndrome (NCS) or mental neuropathy is a sensory neuropathy. It was first described by Charles Bell IN 1830 [1] (BELL C On the nerve of the face, in nervous system and human body: embracing the pears delivered to the royal society subject of the nerves 1833, Duff Green: Washington p65. Google Scholar). It can present as trivial finding of hypoesthesia/paraesthesia or pain over the chin and area supplied by the mandibular nerve. The etiologies of this condition can be grouped as $[2,3]$ :

a) Dental: Iatrogenic trauma during oral surgical procedure (Most common cause). b) Malignancy/Systemic Disease: In the absence of any trauma due to dental/oral surgical procedures NCS has been reported as the first sign of Primary malignancy like osteosarcoma of the mandible and oral squamous cell carcinoma metastatic malignanciesof the breast, lung, thyroid etc. or hematological malignancies such as lymphoma, leukemia, multiple myeloma. It has also been reported to have presented initially as numbness over the chin. This could be considered as herald sign of relapse in patients with history of malignancy (Rogers sign).

c) Neurological: Tumors of the inferior alveolar nerve and the sheaths and compression at the base of the skull by tumor mass may also cause NCS (Figure 3). 

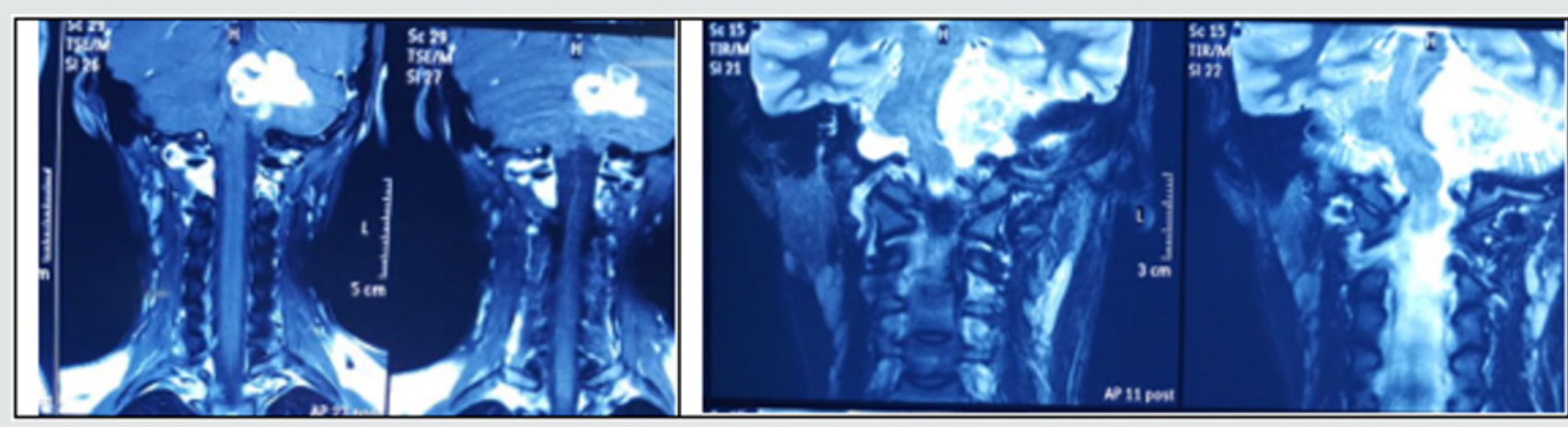

Figure 3: MRI Coronal View.

In the presence of metastatic disease NCS is an advanced symptom. This symptom is often overlooked or not urgently investigated. It is one of the symptoms with poor prognosis, only $15 \%$ of patients are known to survive more than nine months. NCS has better prognosis if it has leptomeningeal involvement. Hence the role of dentists, ENT specialists and physicians is to consider malignancies and neurological disorders in patients with NCS in the absence of local causes. Patients need investigation to rule out malignancies and neurological disorder. This involves blood examination and radiographs which include OPG, brain MRI and targeted imaging such as CT scan and scintigraphy [4]. The trigeminal nerve root originates from the lateral aspect of the pons and extends superiorly, laterally and anteriorly towards the petrous apex entering the Meckel's cave. The first segment which extends from the brainstem to the Meckel's cave is referred to as cisternal or cerebellopontine angle segment. The cerebellopontine angle is a triangular space located posterior to the pyramid inferior to the tentorium, lateral to pons and ventral to cerebellum.

The most common mass found in this location is vestibular schwannoma, commonly referred to as acoustic neuroma, followed by meningioma and epidermoid tumors and rarely metastases [5]. Nerve sheath tumors can be dividing into schwannoma, neurofibroma and malignant nerve sheath tumors. Schwannoma/ neurilemmomas are encapsulated, benign slowly growing neoplasms arising from Schwann cells of central, spinal, peripheral nerves, oligodendrocytes of the myelin in the brain. Primary intracranial nerve sheath neoplasm are mostly schwannomas and they do not have malignant potential. 5- 20\% of these cases are associated with type 2 neurofibromatosis [6]. Type 2 neurofibromatosis is an inherited autosomal dominant syndrome with a propensity for developing multiple schwannomas, meningiomas and gliomas [7,8]. A mutation in the tumor suppressor gene $\mathrm{NF}_{2}$ located on chromosome 22 is identified as the reason for this syndrome. Neurofibroma in contrast, have propensity for malignant transformation. Spinal neurofibroma is sporadic tumor and is usually sign of von Recklinghausen disease i.e., type 1 neurofibramatosis. There are 3 types of neurofibromatosis.

a) Type 1 NF1: Skin and bony changes present early usually in childhood. b) Type 2 NF2: Associated with Hearing loss and poor balance.

c) Type 3 NF3/Schwannomatosis: Congenital neurilemmoma characterized by multiple peripheral nerve tumors without the involvement of vestibular nerve.

Intracranial nerve schwannomas are benign tumors that are slow growing [9]. Most of them arise from cranial nerves, the most common being the vestibular schwannoma. Clinical signs are: Progressive unilateral sensorineural dysacusis, Intermittent hearing loss, Tinnitus and sensation of fullness in the ear, Vertigo, difficulty in walking, Visual blurring, Trigeminal neuralgia and headaches. All these signs were absent in our patient. Trigeminal schwannomas account to only about $0.2 \%-0.8 \%$ of the total intracranial nerve schwannomas it is also associated with neurofibromatosis -2 . Their clinical presentation can vary from neuralgia, neurasthenia or numbness as in our case. It has female predilection. Jefferson has classified trigeminal schwannomas based on their location into four types [10].

CT imaging displays it as is dense regions difficult to differentiate. Areas within large tumors are hypodense. On administration of contrast it shows moderate enhancement. MRI is the investigation of choice for these lesions. T1- weighted image, it appears as isointense and cystic areas appears hypointense. T2 images it appears as hyperintense to brain and cystic areas appear hyperintense [11]. Treatment is directed at symptomatic control. Surgical resection of intracranial tumors carries a risk of significant morbidity such as hydrocephalus and meningitis. Microsurgical techniques have better outcome with preservation of neurologic function. Malignant nerve sheath tumors have poor prognosis [11]. After the neurological evaluation, our patient has been kept under observation, it was considered best not to perform any intervention. Patient has been under continuous follow up and during the preparation of this manuscript she has been found to be stable.

\section{Conclusion}

It is important that symptoms of parathesia over the chin are never overlooked as trivial in patients. Investigations such as MRI of brain may reveal an underlying pathology that may be detrimental. 


\section{References}

1. Smith RM, Hassan A, Robertson CE (2015) Numb Chin Syndrome. Curr Pain Headache Rep 19(9): 44.

2. Maeda K, TaniguchiJI, Matsui K (2018) Two cases of numb chin syndrome diagnosed as malignant disease. Oxf Med Case Reports 12(1): omy097.

3. Lu SY, Huang SH, Chen YH (2017) Numb chin with mandibular pain or masticatory weakness as indicator for systemic malignancy - A case series study. J Formos Med Assoc 116(11): 897-906.

4. James G Smiriniotopoulos, Nancy Chang Yue, Elisabeth J Rusting (1993) Cerebellopontine angle masses: Radiologic-Pathologic Correlataion. Radiographics 13(5): 1131-1147.

5. Lin X, Lee KE (2013) Numb cheek syndrome: A clinical-anatomical correlation. Diagnosis: Trigeminal schwannoma. Eur Neurol 70(5-6): 331-332.

6. Sanabria Sanchinel AA, Flores Robles BJ, Santos Lasaosa S (2016) Numb chin syndrome secondary to solitary schwannoma]. Med Clin (Barc) 47(3): 134 .
7. Rauen KA, Huson SM, Burkitt Wright E, Evans DG, Farschtschi S, et al (2014) Recent developments in neurofibromatoses and RASopathies: Management, diagnosis and current and future therapeutic avenues. Am J Med Genet A 167A (1): 1-10.

8. Ferner RE, Shaw A, Evans DG, Dympna McAleer, Dorothy Halliday, et al. (2014) Longitudinal evaluation of quality of life in 288 patients with neurofibromatosis 2. J Neurol 261(5): 963-969.

9. Dirks MS, Butman JA, Kim HJ, Wu T, Morgan K, et al. (2012) Long-term natural history of neurofibromatosis Type 2-associated intracranial tumors. J Neurosurg 117(1): 109-117.

10. Agarwal A (2015) Intracranial trigeminal schwannoma. Neuroradiol J 28(1): 36-41.

11. Chowdhury FH, Haque MR, Kawsar KA, Sarker MH, Hasan M, et al. (2014) Intracranial non vestibular neurinomas: Young neurosurgeons' experience. J Neurosci Rural Pract 5(3): 231-243.

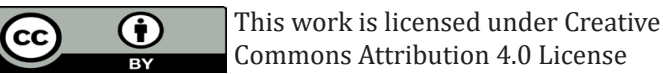

To Submit Your Article Click Here: Submit Article

DOI: $10.32474 /$ SJO.2019.01.000120

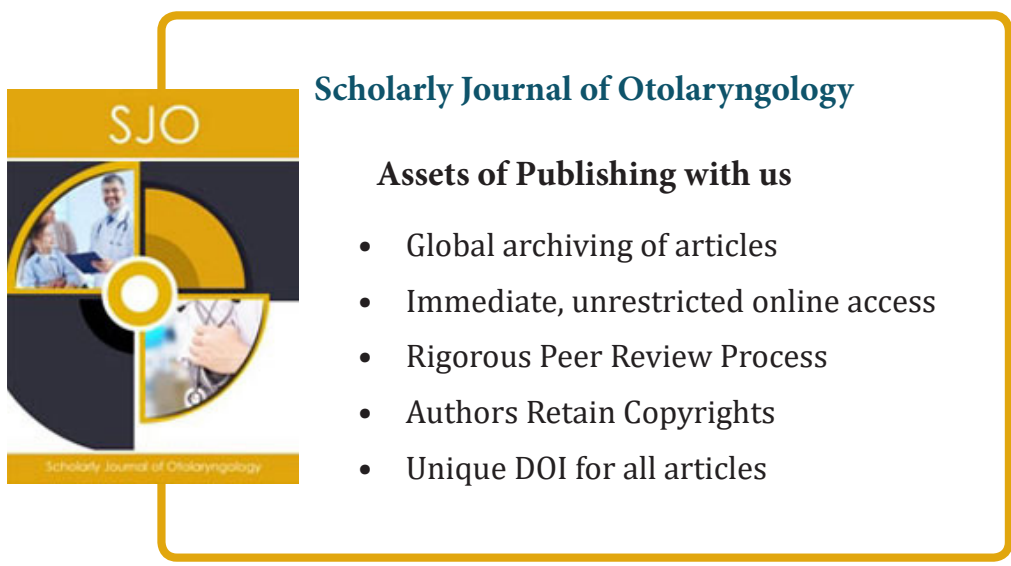

